\title{
Laminar separation bubbles: Dynamics and control
}

\author{
SOURABH S DIWAN and O N RAMESH \\ Department of Aerospace Engineering, Indian Institute of Science, \\ Bangalore 560012 \\ e-mail: \{diwan,onr\}@aero.iisc.ernet.in
}

\begin{abstract}
This work is an experimental investigation of the dynamics and control of the laminar separation bubbles which are typically present on the suction surface of an aerofoil at a large angle of attack. A separation bubble is produced on the upper surface of a flat plate by appropriately contouring the top wall of the wind tunnel. First, a basic (unforced) separation bubble is obtained to set a benchmark for further experiments. Parametric study is done where the reference velocity is decreased to quantify its effect on the aspect ratio of the bubble. It is found that with decrease in Reynolds number, the height of the bubble increases at a greater rate than the length. This feature could be useful in characterising separation bubbles especially from the point of view of low Reynolds number aerofoil design. Artificial disturbance is introduced at two different initial amplitudes (infinitesimal and finite) upstream of separation location and hotwire anemometry is used to trace the wave packet as it is advected downstream. The evolution of wave packets is seen to take place in two distinct stages. Finite amplitude forcing causes periodic quenching of the bubble. Interestingly, even an infinitesimally small forcing is seen to modify and thereby control the separation bubble.
\end{abstract}

Keywords. Laminar separation bubble; wave packet; external excitation; control.

\section{Introduction}

Under the influence of an adverse pressure gradient, a laminar boundary layer separates from the surface, becomes transitional and ultimately the separated shear layer reattaches to form a 'laminar separation bubble'. Such bubbles are typically observed close to the leading edges of thin aerofoils, on gas turbine blades and on low Reynolds number micro-aero-vehicle wings. Presence of bubbles has a deteriorating effect on the performance of the device. The understanding of the physics of the laminar separation bubble and possible ways to control it thus are essential prerequisites for efficient design of these aerodynamic devices. Gaster (1967) was the first to systematically explore the stability characteristics associated with the transition taking place in separation bubble. Many recent studies have been directed towards exploring the dynamics of separation bubbles (see Watmuff 1999, Pauley et al 1990, Marxen et al 2003).

The bubble constitutes, at the same time, laminar separation, transition to turbulence and aspects of both the attached as well as free shear layer. Even though there is a fair amount 
of understanding of the individual aspects, the interplay amongst them is far from being understood. However, there are some gross features exhibited by the bubble which can be discerned from measurements. In the present work, it is intended to study the overall bubble characteristics. The global properties of the bubble include the length, height and shape of the bubble, the pressure distribution on the plate, the amplification rates of disturbances through the bubble etc. This is the first essential step towards gaining better understanding of the structure of the bubble.

In order to control the separation bubble, Howell (1999) introduced an artificial turbulent spot (by introducing high intensity disturbance impulsively) upstream of the bubble and studied its effect on the loss reduction in a gas turbine blade due to periodic quenching of the separation bubble. On the other hand, Watmuff (1999) impulsively forced the separation bubble with small amplitude disturbances for exploring the instability characteristics. However, the possible role of small amplitude impulsive forcing in controlling the size of the separation bubble and hence deciding the optimum amplitude so as to minimize the energy expenditure was not addressed in these studies. Diwan (2005) extensively studied the evolution of finite as well as infinitesimal wave packets through the separation bubble with a view towards its control. In the present paper, we discuss the role of finite as well as infinitesimally small disturbances in modifying the size of the bubble. Building block experiments have been carried out to understand the above stated methodology for controlling the separation bubble.

\section{Experimental setup}

The present experiments have been conducted in a closed circuit wind tunnel ( $4 \mathrm{~m}$ long and $1 \mathrm{~m} \times 1 \mathrm{~m}$ in cross-section) at the Indian Institute of Science. The turbulence intensity was measured to be less than $0.03 \%$. The experimental setup is shown in figure 1 .

Experiments have been carried out on the upper surface of a flat plate mounted in the test section. An adverse pressure gradient was imposed on the plate by appropriately contouring the top wall. Single component stationary hotwire anemometry was employed for velocity measurements. Surface and smoke flow visualisation techniques were employed to measure the length and average height (maximum height of the zero mean line) of the bubble which were compared with the ones obtained from velocity measurements.

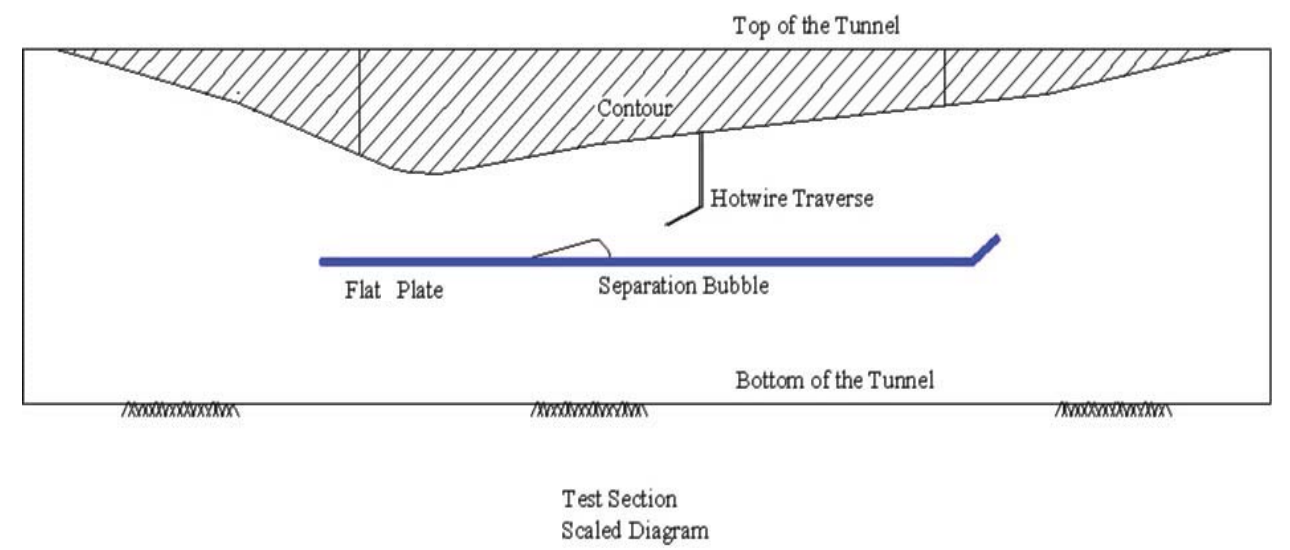

Figure 1. The experimental setup. 
Artificial disturbance was introduced into the flow through one of the pressure ports using a loudspeaker. The loudspeaker was driven by an electronic driver developed in-house which supplied square waves of narrow width $(10 \mathrm{~ms})$ at regular intervals to the loudspeaker.

Experiments have been conducted using different settings of the top contour and imposing various pressure distributions on the plate. Figure 1 shows a typical experimental arrangement to achieve the required pressure distribution on the flat plate; two slightly different top roof configurations (to be called pressure settings $A$ and $B$ ) have been used in the experiments.

\section{Results}

\subsection{The basic (unforced) separation bubble}

Experiments on unforced separation bubbles have been done for a reference speed $U_{\text {ref }}=$ $5.46 \mathrm{~m} / \mathrm{s}$ and pressure setting $A$. The reference speed was measured upstream of the plate using pitot-static tube. The pressure distribution along the centreline of the plate is shown in figure 2 . The coefficient of pressure is given by

$$
C p=\left(P_{x}-P_{\text {inf }}\right) /\left(P_{\text {total }}-P_{\text {inf }}\right),
$$

where, $\quad P_{x}=$ pressure at the desired streamwise location,

$P_{\text {total }}=$ the total pressure measured by pitot-static tube upstream of plate,

$P_{\text {inf }}=$ the reference pressure. Here the static pressure upstream of plate.

' $S$ ' and ' $R$ ' indicate the separation and reattachment points respectively. The separation bubble is characterised by a region of relatively constant pressure followed by a sharp pressure recovery. The streamwise location of maximum height roughly corresponds to the end of constant pressure region (see Tani 1964, Gaster 1967).

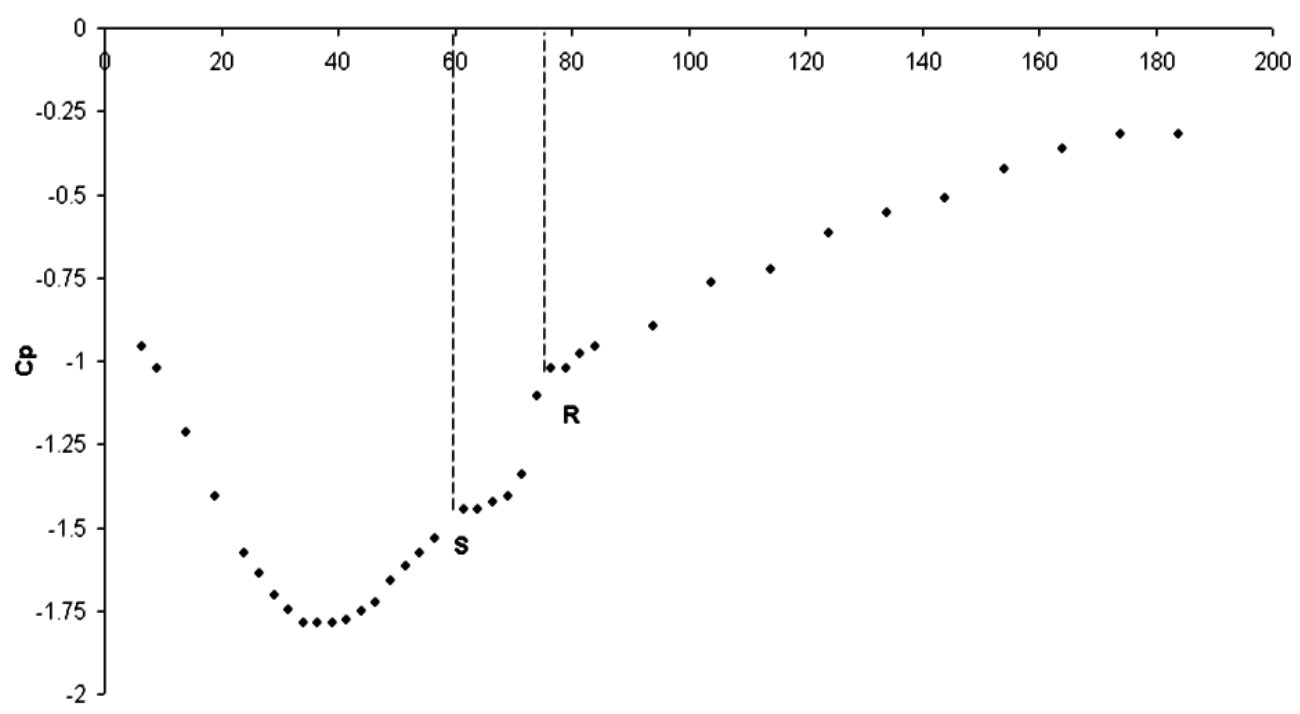

Distance from Leading Edge (cm)

Figure 2. The coefficient of pressure distribution for pressure setting $A$ and $U_{\text {ref }}=5.46 \mathrm{~m} / \mathrm{s}$. 


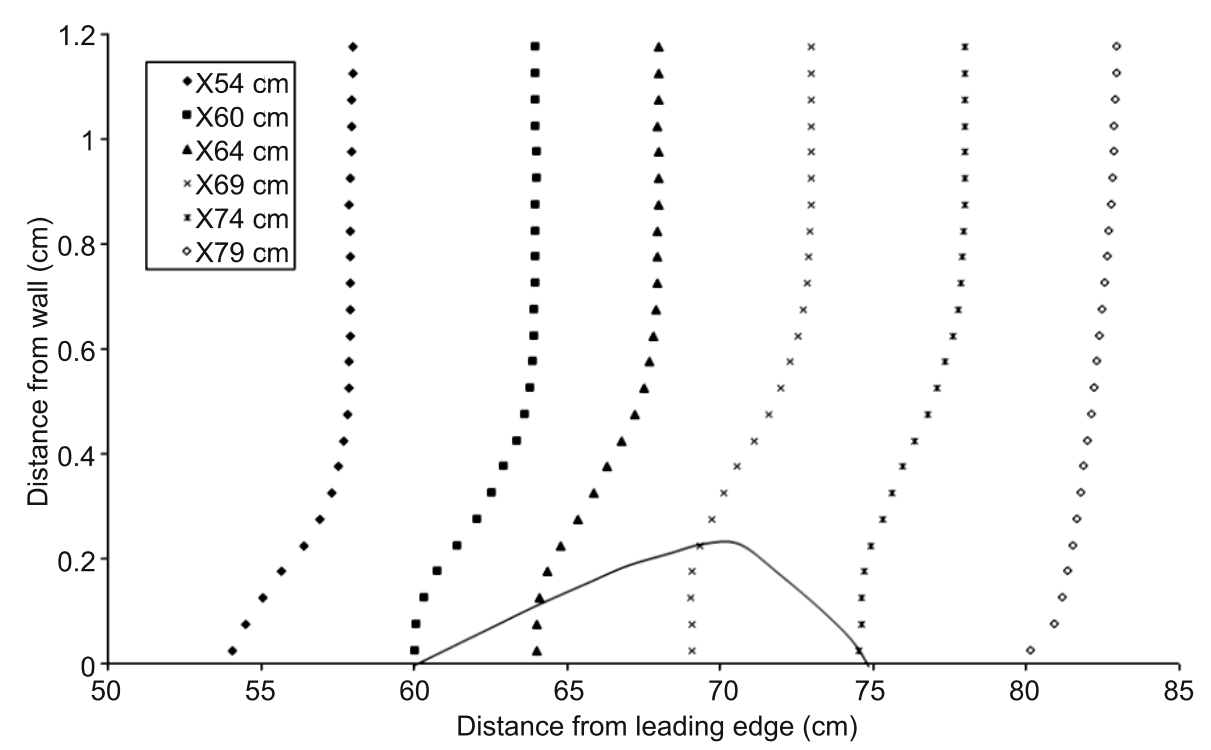

Figure 3. The normalised mean velocity profiles at different streamwise locations for pressure setting $A$ and $U_{\text {ref }}=5.46 \mathrm{~m} / \mathrm{s}$. The solid line represents the bubble outline.

Figure 3 shows the normalised mean velocity profiles in relation to the time-averaged bubble outline at different streamwise locations for $U_{\text {ref }}=5.46 \mathrm{~m} / \mathrm{s}$ and pressure setting $A$. From smoke flow visualisation the height of the bubble was measured to be roughly $2.3 \mathrm{~mm}$. Figure 4 shows a typical smoke flow visualisation picture. The Reynolds number based on the freestream velocity and the momentum thickness at separation was calculated to be 551.4.

\subsection{Variation of bubble size with decreasing reference speed}

The contoured top wall was kept unaltered as shown in figure 1 and experiments were done for different reference speeds (in descending order) to see its effect on height and length of the bubble. It is seen from the pressure distribution and the smoke flow visualisation that both the length and height of the bubble increase with decrease in reference speed. Perhaps the most appropriate parameter to quantify the size of the bubble is the aspect ratio $h / L$. It also is an indication of the geometric bluffness of the bubble. The aspect ratio is plotted against Reynolds number $\left(\operatorname{Re}_{\theta s}\right)$ and is depicted in figure 5 which includes some of the data available in the literature.

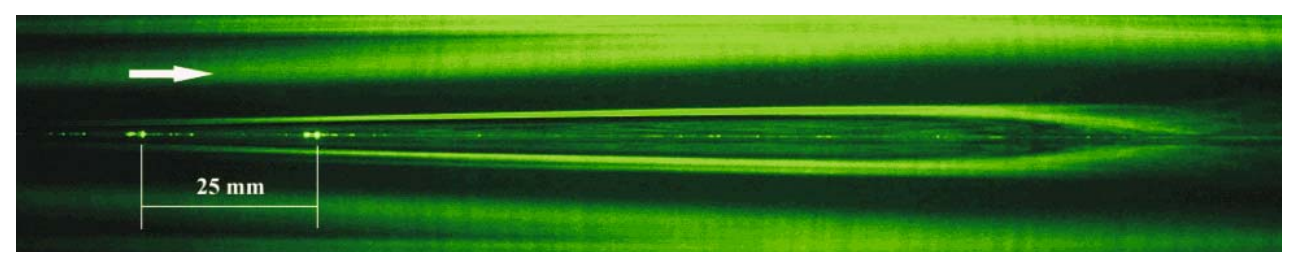

Figure 4. A typical smoke flow visualisation picture depicting the separation bubble. The flow is from left to right. Also visible is the reflection of the bubble in the polished surface of the plate. 


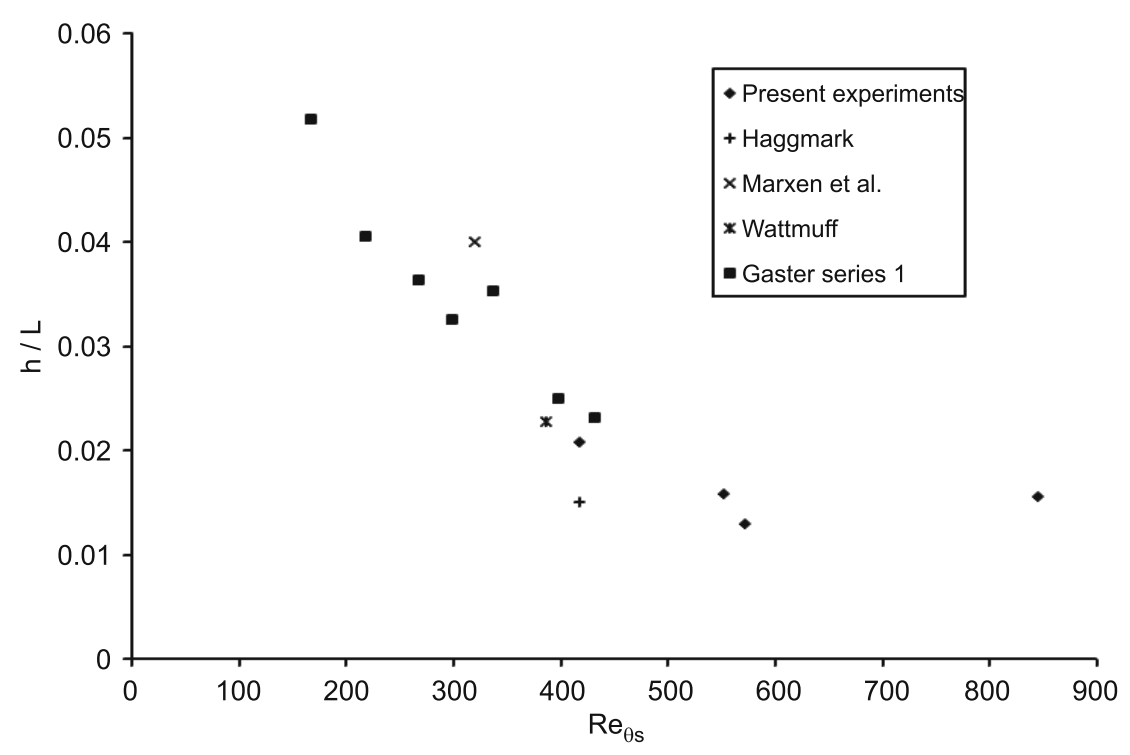

Figure 5. The variation of aspect ratio of the bubble with the Reynolds number for different bubbles. Pressure setting $A$.

It shows a clear cut trend with a reasonable collapse where the aspect ratio goes on increasing with decreasing Reynolds number. The decrease in Reynolds number causes increase both length as well as height. However, to satisfy the inverse relation of the aspect ratio with the Reynolds number (as seen in figure 5), the relative increase in height $(d h / h)$ must be greater than the relative increase in length $(d L / L)$. It is thus apparent from the above discussion that the height increases at a greater rate with decrease in Reynolds number compared to the corresponding variation of length.

\subsection{Response of the bubble to external excitation}

An impulsive disturbance was introduced into the boundary layer upstream of separation at the location of minimum pressure. Two different amplitudes were used, $S 1$ and $S 2$, at increasing levels of intensity to study the response of the bubble to infinitesimal as well as finite amplitude disturbances. The impulse response of the flow was monitored along the line joining the maximum RMS intensity (for the unexcited separation bubble) which was seen to correspond to the line joining the inflection points in the mean velocity profiles for the most part of the bubble. The interval between the pulses was 1 second, and phase averaging was done over 128 pulses. Figure 6 shows the evolution of disturbances in the streamwise direction for pressure setting $B, U_{\text {ref }}=5.46 \mathrm{~m} / \mathrm{s}$ and the amplitude $S 1$ (infinitesimal disturbance).

The impulsively added disturbance forms a wave packet, which is advected downstream by the flow. As it travels downstream it is dispersed and there is also a change in its structure. This downstream development of the wave packet can be divided into two stages based on the linearity of the leading and trailing edges of the wave packet. The switchover from one stage to another takes place at a location a little upstream of the location of the maximum height $(M)$ shown by the broken line signal in figure 6 .

A summarised version plot of the effect of change in the initial amplitude level on the streamwise variation of RMS intensity is plotted in figure 7. It is seen that the finite amplitude 


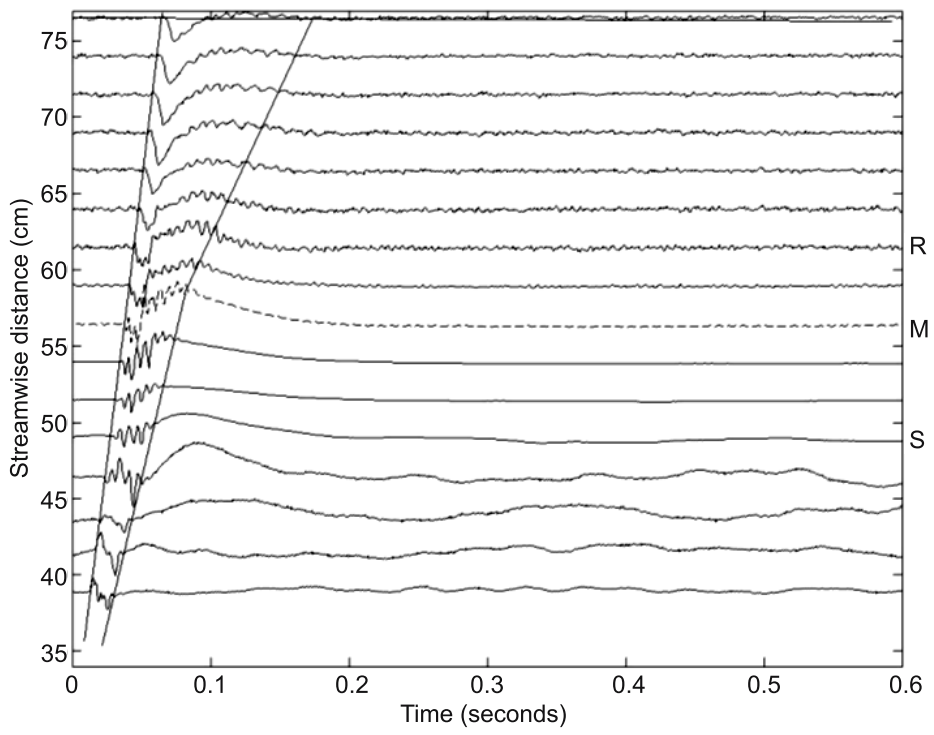

Figure 6. The evolution of wave packets in the streamwise direction for pressure setting $B$, forcing amplitude $S 1$ and $U_{\text {ref }}=5.46 \mathrm{~m} / \mathrm{s}$.

forcing such as $S 2$ substantially alters the shape of the bubble as can also be seen by the elevated levels of RMS intensity for $S 2$ in the initial portion of the bubble in figure 7. It is interesting to note that even an infinitesimal forcing could modify or control the laminar separation bubble structure. Perhaps the instantaneous velocity profile arising from infinitesimal forcing could

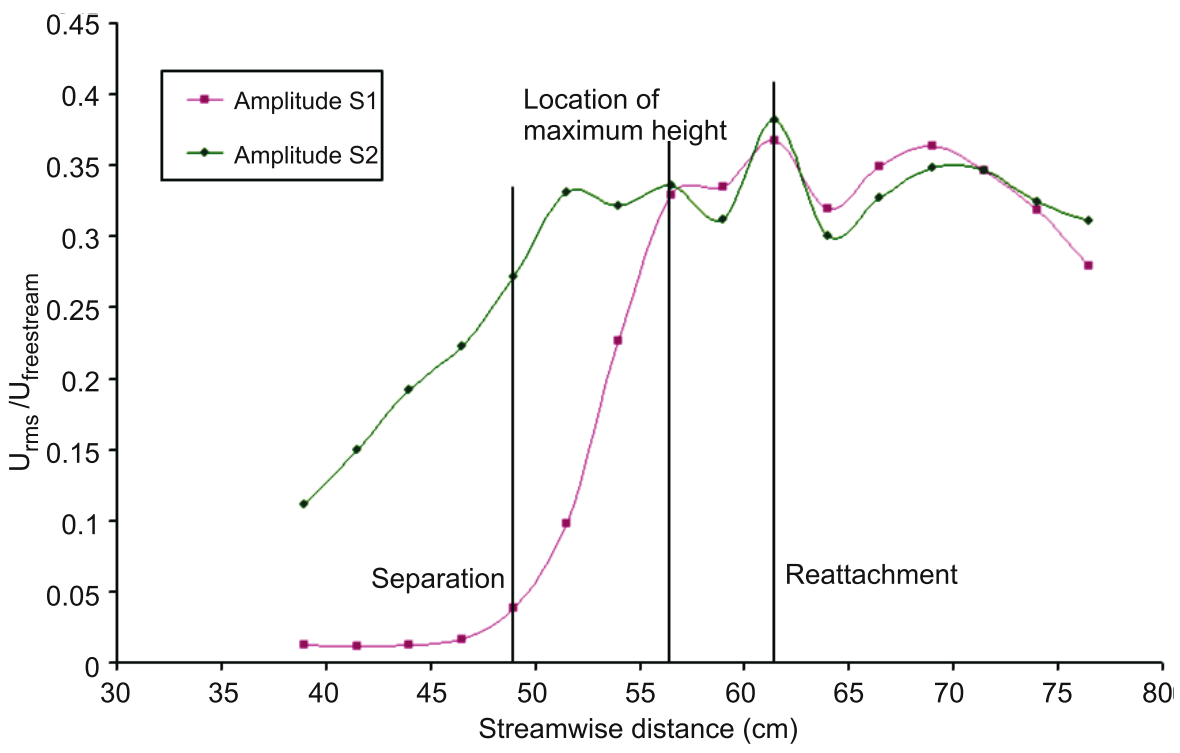

Figure 7. The variation of RMS intensity in the streamwise direction for amplitude settings $S 1$ and $S 2$. Pressure setting $B$ and $U_{\text {ref }}=5.46 \mathrm{~m} / \mathrm{s}$. 
be imagined to energise the retarded dead air fluid in the front portion of the bubble thereby making the velocity profile fuller and therefore more stable.

\section{Conclusion}

A laminar separation bubble was produced experimentally on a flat plate and the basic structure was established. The effect of reduction in reference speed on the bubble aspect ratio (ratio of height to length of the bubble) was studied, showing that both length and height of the bubble increase with decrease in speed. However, further analysis shows that the height increases at a greater rate than the length. This feature could be useful in characterising separation bubbles, especially from the point of view of low Reynolds number aerofoil design.

Tracking of the externally introduced wave packet through the separation bubble shows that the evolution can be split into two stages based on the linear envelope of the leading and trailing edges of the wave packet. With finite amplitude forcing, the shape and size of the bubble was seen to alter substantially with periodic quenching of the bubble. Interestingly, even an infinitesimally small forcing was seen to modify and thereby control the separation bubble.

We dedicate this paper to Dr P R Viswanath on the occasion of his sixtieth birthday.

\section{References}

Diwan S S 2005 Response of a laminar separation bubble to external excitation. M Sc(Engg) thesis, Indian Institute of Science, Bangalore

Gaster M 1967 The structure and behaviour of separation bubbles. ARC R\&M 3595

Haggmark C P, Bakchinov A A, Alfredson P H 2000 Experiments on a two-dimensional laminar separation bubble. Philos. Trans. R. Soc. London A358: 3193-3205

Howell R J 1999 Wake-separation bubble interactions in low Reynolds number turbomachinery, Ph D thesis, Cambridge University Engineering Department, Cambridge, UK

Marxen O, Lang M, Rist U, Wagner S A 2003 Combined experimental/ numerical study of unsteady phenomena in a laminar separation bubble. Flow, Turbulence Combustion 71: 133-146

Pauley L L, Moin P, Reynolds W C 1990 The structure of two-dimensional separation. J. Fluid Mech. 220: 397-411

Tani I 1964 Low speed flows involving bubble separations. Prog. Aerosp. Sci. 5: 70-103

Watmuff J H 1999 Evolution of a wave packet into vortex loops in a laminar separation bubble. J. Fluid Mech. 397: 119-169 